\title{
Treatment of Pulmonary Arterial Hypertension: Great Expectations!
}
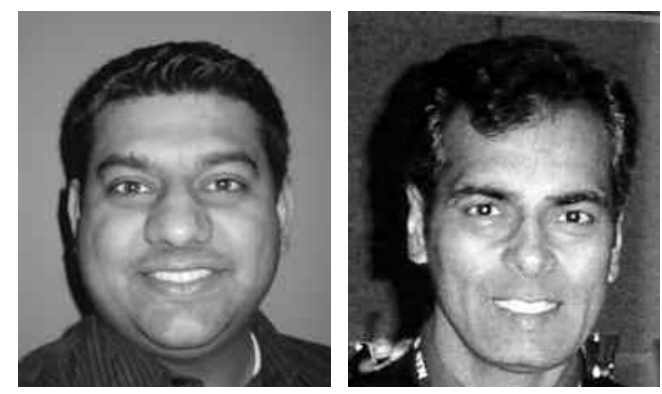

"Take nothing on its looks; take everything on evidence. There's no better rule."

\section{- Charles Dickens, Great Expectations}

Pulmonary arterial hypertension (PAH) is a serious, progressive, often fatal condition characterized by increased pulmonary artery pressure (PAP) and pulmonary vascular resistance $(\mathrm{PVR})^{1,2}$. Patients with PAH experience dyspnea and exercise limitation - and as right ventricular (RV) failure develops, edema, exertional chest pain, syncope, and increased risk of death. PAH is most commonly idiopathic (IPAH) or associated with an underlying connective tissue disease (CTD-PAH), such as scleroderma ${ }^{1}$. In both IPAH and CTD-PAH patients, median survival is less than 3 years in the absence of therapy ${ }^{3,4,5}$.

Research advances in our understanding of the pathobiology of PAH have led to the study, approval, and availability of many novel PAH-specific medications. These include prostanoid analogs (i.e., intravenous epoprostenol, subcutaneous/intravenous treprostinil), endothelin receptor antagonists (i.e., ambrisentan, bosentan), and phosphodiesterase type-5 inhibitors (i.e., sildenafil, tadalafil) ${ }^{2,6}$.

What are the expectations of this array of therapies on the part of patients with PAH and their physicians? The published evidence, typically short-term, 3 to 4-month, randomized controlled trials of all these therapies, demonstrates benefit in both IPAH and CTD-PAH ${ }^{7}$. A majority of patients have small reductions in PAP and PVR, increased cardiac output, decreased symptoms, and increased exercise capacity, as measured by 6-minute walk test (6-MWT). New York Heart Association/World Health Organization (NYHA/WHO) functional class will improve in about one-third of patients, and objectively measured quality of life (QOL) may also improve ${ }^{2,6,7}$. A metaanalysis of published randomized controlled trials of PAH-specific therapies also confirmed a small, short-term survival benefit ${ }^{7}$.

Thus, based on the evidence, patients with PAH and their physicians expect symptomatic benefit and improved functional capacity, important components of improved QOL. However, in a progressive, fatal disease like PAH, short-term benefits are not enough; patients also expect longterm improvement in clinically significant outcomes, such as prolonged survival, and less need for intensification of therapy, including hospitalization or lung transplantation.

The potential for longterm benefit of many current PAH-specific therapies with regard to functional capacity and NYHA/WHO class, and possibly even better survival, has been suggested by reports of open-label trials and clinical experience. However, as the prognosis for survival in an individual patient with PAH is difficult to predict, conclusions about improved survival with PAH-specific therapies are usually based on the natural history of untreated IPAH patients in the US National Institutes of Health (NIH) registry of 194 IPAH patients followed from 1981 to $1985^{3}$. The NIH registry identified 3 hemodynamic variables (mean PAP, right atrial pressure, and cardiac index) predictive of survival in multivariate analysis, and developed a survival predictive equation. This NIH survival equation has been widely used to estimate survival of IPAH patients in clinical practice and in clinical trials.

However, the NIH survival equation may not apply to IPAH patients in the current treatment era, because of recognized changes in demographics (e.g., generally older) and clinical characteristics (e.g., more comorbid illnesses) of contemporary IPAH patients, and potential effects of background therapies, such as diuretics and supplemental oxygen. Moreover, the NIH survival equation only ever applied to IPAH patients, providing no information on the longterm prognosis of other PAH patients, such as CTD-PAH.

Besides pulmonary hemodynamics, many other "surrogate" endpoints have been recognized as important prognostic factors in PAH. These include clinical features such as age, sex, type of PAH, and the degree of clinical limita-

See Effect of warfarin on survival in SSc-PAH and IPAH, page 462

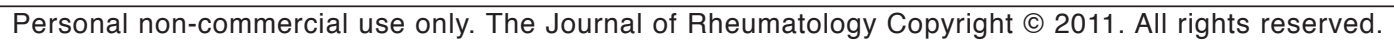


tion (e.g., NYHA/WHO functional class). Objective laboratory measurements also correlate with survival, including exercise capacity (e.g., 6-MWT), echocardiographic measures of RV dysfunction (e.g., Tei index, pericardial effusion), and plasma mediator levels (e.g., brain natriuretic peptide).

In an individual patient with $\mathrm{PAH}$ treated with a new PAH-specific medication, how do we assess whether expectations are met? Which of the broad panel of surrogate outcomes should be assessed to indicate improvement in the severity of PAH? Is a measurable change in a single endpoint (e.g., greater 6-MWT distance) enough to satisfy our expectations that a treatment is of benefit to the patient? Most important, do short-term improvements in surrogate outcomes reasonably provide expectations of better longterm clinical stability and survival? Although we do not have the answers to most of these questions, many new initiatives are addressing these and other fundamental questions in the clinical management of patients with PAH.

One such initiative is the REVEAL registry, a collaborative effort of 54 US PAH centers, which has enrolled almost 3000 PAH patients to better characterize clinical and treatment aspects of PAH patients in the modern era ${ }^{8,9}$. In 2716 PAH patients followed over one year, 15 key demographic (e.g., age), clinical (e.g., type of PAH, NYHA/WHO functional class), hemodynamic (e.g., PVR), and laboratory (e.g., 6-MWT distance, brain natriuretic peptide) independent predictors of survival were identified in multivariable analysis ${ }^{9}$. The results emphasize the importance of using the full spectrum of available clinical data for the regular assessment of PAH patients, rather than relying on a single or a few parameters. Practically, a contemporary, multivariable, weighted prognostic equation was formulated for use during regular PAH patient evaluation in order to predict individual prognosis, and to allow individualization and optimization of treatment approaches.

As physicians, our expectations of the benefits of PAH medical therapies are presumably based on evidence, but personal experience and beliefs also may contribute. In this issue of The Journal, Johnson, et al surveyed a group of $\mathrm{PAH}$ experts and quantified their expectations of the survival benefit of warfarin ${ }^{10}$. They accomplished this through Bayesian inference, an analytic method that incorporates experts' beliefs about the effect of an intervention into models estimating treatment effect by expressing these beliefs as prior probability distributions.

Overall, PAH experts believed warfarin improved survival, but their expectation of survival benefit over 3 years was at best small (2\% in scleroderma-PAH and $8 \%$ in IPAH). There was also considerable uncertainty in the expected magnitude of benefit. Strikingly, the expected benefit was much lower than the $20 \%$ survival benefit found in Johnson's metaanalysis of 4 published studies of warfarin therapy in IPAH, and the benefits reported in a previous qualitative systematic review of 7 observational studies ${ }^{11}$. Warfarin use has not specifically been studied in CTD-PAH or scleroderma-PAH, and thus, there are no data on the potential benefits, such as survival, of anticoagulation in scleroderma-PAH. Thus, our own expectations may not be consistent with published evidence. Indeed, despite the limited expectation of benefit, $84 \%$ of PAH experts surveyed admitted to routine use of anticoagulation in both IPAH and scleroderma-associated-PAH ${ }^{10}$. Similarly, $53.4 \%$ of 2716 PAH patients in the REVEAL registry were currently treated with warfarin, including $42.6 \%$ of CTD-PAH patients ${ }^{8}$.

Our expectations of the benefits of PAH-specific therapies in individual PAH patients also depend on other factors, including the type of PAH. Johnson, et al importantly highlight medical community uncertainty about the therapeutic approach to different types of PAH, specifically IPAH versus CTD-PAH ${ }^{10}$. In the REVEAL registry, CTD-PAH patients were older, more likely female, and more likely African American than IPAH patients ${ }^{12}$. Patients with CTD-PAH have more severe clinical disease than IPAH patients, characterized by more dyspnea, poorer functional capacity, and greater mortality despite less severe pulmonary hemodynamics and less echocardiographic RV dysfunction $4,5,8,9,13,14$. The REVEAL registry specifically identified CTD-PAH as an important adverse prognostic factor, with a hazard ratio for death of 1.59 relative to IPAH ${ }^{9}$.

Despite expectations, surrogate endpoints may not be as prognostically useful in the assessment of CTD-PAH patients as in IPAH patients. The 6-MWT has not been specifically validated in CTD-PAH, and there are many potential confounding clinical factors in these patients that could affect both the results and the ability of the patient to perform the test. These include conditions associated with CTD, such as interstitial lung disease and musculoskeletal manifestations, as well as comorbid cardiac disease ${ }^{15}$. Several studies have reported lower 6-MWT distance in CTD-PAH compared to IPAH patients, despite less severe PAH by hemodynamic criteria ${ }^{12,13,14}$

Finally, CTD-PAH patients often have a less favorable response to current PAH-specific therapies ${ }^{4,9,16}$. For example, patients with scleroderma-PAH treated with epoprostenol had more than twice the mortality rate of similarly treated patients with IPAH ${ }^{16}$. A possible explanation for the poorer outcomes in CTD-PAH patients may be less aggressive PAH treatment than IPAH patients, as shown in REVEAL $^{12}$. CTD-PAH patients were less likely to be treated with prostacyclin and multiple PAH-specific therapies in "combination." Despite the recognized poor prognosis of patients with CTD-PAH, physicians' own negative expectations of less favorable responses to $\mathrm{PAH}$ therapies or increased risks of these complex, aggressive therapies may result in a hesitancy to initiate them.

Over the past 15 years, the expectations of PAH patients and their physicians have evolved dramatically. From a

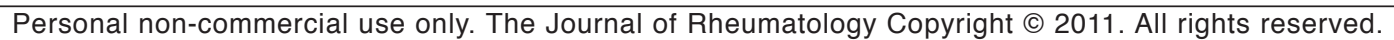


nearly universally fatal disease, the clinical course of PAH has changed for many patients to a chronic condition that can be managed with a broad array of effective PAH-specific therapies. Expectations for improved clinical well being and QOL - and likely also longer survival - are currently being met. However, expectations persist regarding more effective treatment approaches, more evidence-based, rather than belief-based, rational use of medications, and possibly a cure.

GANESH SUBRAMANIAN, MD, FRCPC,

Department of Medicine,

University of Alberta,

Edmonton, Alberta;

SANJAY MEHTA, MD, FRCPC FCCP

Director, Southwest Ontario Pulmonary Hypertension Clinic,

Division of Respirology,

Department of Medicine,

London Health Sciences Center;

Professor, Program in Critical Illness Research,

Lawson Health Research Institute,

University of Western Ontario,

London, Ontario, Canada

Address correspondence to Dr. S. Mehta, Division of Respirology, London Health Sciences Center, Victoria Hospital, Room E1.325, 800 Commissioner's Road East, London, ON N6A 5W9, Canada. E-mail: sanjay.mehta@lhsc.on.ca

Dr. Mehta has received consulting and speaking fees (Actelion, Bayer, GSK, Pfizer) and clinical investigator fees (Actelion, Bayer, Gilead, GSK, Lilly, Pfizer, United Therapeutics).

\section{REFERENCES}

1. Simonneau G, Robbins IM, Beghetti M, Channick RN, Delcroix M, Denton CP, et al. Updated clinical classification of pulmonary hypertension. J Am Coll Cardiol 2009;54 Suppl:S43-54.

2. Galie N, Hoeper MM, Humbert M, Torbicki A, Vachiery JL, Barbera JA, et al. Guidelines for the diagnosis and treatment of pulmonary hypertension: The Task Force for the Diagnosis and Treatment of Pulmonary Hypertension of the European Society of Cardiology (ESC) and the European Respiratory Society (ERS), endorsed by the International Society of Heart and Lung Transplantation (ISHLT). Eur Heart J 2009;30:2493-537.

3. D'Alonzo GE, Barst RJ, Ayres SM, Bergofsky EH, Brundage BH, Detre KM, et al. Survival in patients with primary pulmonary hypertension. Results from a national prospective registry. Ann Intern Med 1991;115:343-9.
4. Hesselstrand R, Wildt M, Ekmehag B, Wuttge DM, Scheja A. Survival in patients with pulmonary arterial hypertension associated with systemic sclerosis from a Swedish single centre: prognosis still poor and prediction difficult. Scand J Rheumatol 2010 Sep 21 [epub ahead of print].

5. Koh ET, Lee P, Gladman DD, Abu-Shakra M. Pulmonary hypertension in systemic sclerosis: an analysis of 17 patients. Br J Rheumatol 1996;35:989-93.

6. Barst RJ, Gibbs JS, Ghofrani HA, Hoeper MM, McLaughlin VV, Rubin LJ, et al. Updated evidence-based treatment algorithm in pulmonary arterial hypertension. J Am Coll Cardiol 2009;54 Suppl:S78-84.

7. Galie N, Manes A, Negro L, Palazzini M, Bacchi-Reggiani ML, Branzi A. A meta-analysis of randomized controlled trials in pulmonary arterial hypertension. Eur Heart J 2009;30:394-403.

8. Badesch DB, Raskob GE, Elliott CG, Krichman AM, Farber HW, Frost AE, et al. Pulmonary arterial hypertension: baseline characteristics from the REVEAL registry. Chest 2010;137:376-87.

9. Benza RL, Miller DP, Gomberg-Maitland M, Frantz RP, Foreman AJ, Coffey CS, et al. Predicting survival in pulmonary arterial hypertension: insights from the Registry to Evaluate Early and Long-Term Pulmonary Arterial Hypertension Disease Management (REVEAL). Circulation 2010;122:164-72.

10. Johnson SR, Granton JT, Tomlinson GA, Grosbein HA, Hawker GA, Feldman BM. Effect of warfarin on survival in SSc-PAH and IPAH. Belief elicitation for Bayesian priors. J Rheumatol 2011;38:462-9.

11. Johnson SR, Mehta S, Granton JT. Anticoagulation in pulmonary arterial hypertension: a qualitative systematic review. Eur Respir J 2006;28:999-1004.

12. Chung L, Liu J, Parsons L, Hassoun PM, McGoon M, Badesch D, et al. Characterization of connective tissue disease associated pulmonary arterial hypertension from the REVEAL registry: identifying systemic sclerosis as a unique phenotype. Chest 2010 May 27 [epub ahead of print].

13. Fisher MR, Mathai SC, Champion HC, Girgis RE, Housten-Harris T, Hummers L, et al. Clinical differences between idiopathic and scleroderma-related pulmonary hypertension. Arthritis Rheum 2006;54:3043-50.

14. Kawut SM, Taichman DB, Archer-Chicko CL, Palevsky HI, Kimmel SE. Hemodynamics and survival in patients with pulmonary arterial hypertension related to systemic sclerosis. Chest 2003;123:344-50.

15. Garin MC, Highland KB, Silver RM, Strange C. Limitations to the 6-minute walk test in interstitial lung disease and pulmonary hypertension in scleroderma. J Rheumatol 2009;36:330-6.

16. Kuhn KP, Byrne DW, Arbogast PG, Doyle TP, Loyd JE, Robbins IM. Outcome in 91 consecutive patients with pulmonary arterial hypertension receiving epoprostenol. Am J Respir Crit Care Med 2003; 167:580-6.

J Rheumatol 2011;38:403-5; doi:10.3899/jrheum.101250 